
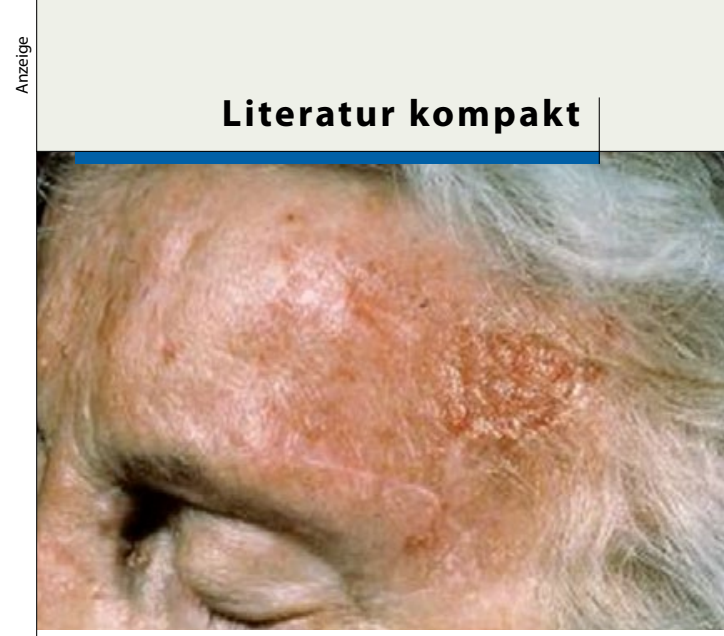

Patient mit Basaliom an der linken Schläfe, fotografisch sauber dokumentiert

\title{
OP nach Hautbiopsie - mit Fotos Fehler vermeiden
}

Treten Patienten nach einer positiven Hautbiospie zur dermatochirurgischen Operation an, ist die fragliche Läsion oft nicht mehr ohne Weiteres zu erkennen. Fotografien der biopsierten Hautstelle würden weiterhelfen, doch die sind leider nur selten verfügbar, wie eine aktuelle Publikation zeigt.

Rund 14\% der Klagen wegen fehlerhafter Behandlung durch Rchirurgische Eingriffe nach Mohs (mikroskopisch kontrolliert, kleiner Sicherheitsabstand) betreffen Operationen an der falschen Stelle. Shari Nemeth von der Mayo Clinic in Scottsdayle und Naomi Lawrence von der Cooper University in Marlton haben nach möglichen Ursachen für diese Art von Fehler gesucht. Dafür schickten sie 722 Mitgliedern des American College of Mohs Surgery einen Fragebogen zu. Die Befragten sollten Auskunft über ihre Probleme geben, die zuvor andernorts biopsierte und nun zu entfernende Hautläsion korrekt zu identifizieren. 325 Kollegen sandten ihre Antworten zurück.

Nur $23 \%$ der befragten Dermatochirurgen berichteten, für mehr als ein Viertel ihrer Patienten wenigstens ein ausreichend genaues Diagramm zu erhalten, in das die zu entfernende Läsion eingezeichnet ist. Lediglich $22 \%$ gaben an, bei mehr als jedem vierten Patienten vor der Operation ein Foto zu erhalten, auf dem die betreffende Hautstelle abgebildet ist.

$71 \%$ der Operateure sagten, mehr als $5 \%$ ihrer Patienten hätten Probleme, die biopsierte Stelle am Tag der Operation zu identifizieren. $10 \%$ der Eingriffe am falschen Ort gehen auf solche irrigen Angaben von Patienten oder ihren Angehörigen zurück. Viele der Patienten haben Mehrfachbiospien hinter sich und können sich nicht erinnern, an welcher Stelle genau sich der positive Befund ergeben hat.

Fazit: Die Angaben der überweisenden Ärzte oder der Patienten sind oft unzuverlässig. Die Lösung des Problems: Der Arzt, der die Biopsie durchführt, sollte die fragliche Region fotografisch dokumentieren. „Der Fotoapparat ist das Röntgengerät des Dermatologen", schreiben Nemeth und Lawrence. $89 \%$ der befragten Operateure betonten, ein gutes Foto sei für die Identifikation der Biopsiestelle die beste Information.

Dr. Robert Bublak

Nemeth SA, Lawrence N. Site identification challenges in dermatologic surgery: A physician survey: J Am Acad Dermatol 2012; May 3 [Epub ahead of print] 